\title{
Application of Neural Network Modeling to Identify Auditory Processing Disorders in School-Age Children
}

\author{
Sridhar Krishnamurti \\ Auburn University, Auburn, AL 36849, USA \\ Correspondence should be addressed to Sridhar Krishnamurti; krishsr@auburn.edu
}

Received 29 August 2014; Revised 4 February 2015; Accepted 5 February 2015

Academic Editor: Paolo Gastaldo

Copyright ( 2015 Sridhar Krishnamurti. This is an open access article distributed under the Creative Commons Attribution License, which permits unrestricted use, distribution, and reproduction in any medium, provided the original work is properly cited.

P300 Auditory Event-Related Potentials (P3AERPs) were recorded in nine school-age children with auditory processing disorders and nine age- and gender-matched controls in response to tone burst stimuli presented at varying rates (1/second or $3 /$ second) under varying levels of competing noise $(0 \mathrm{~dB}, 40 \mathrm{~dB}$, or $60 \mathrm{~dB}$ SPL). Neural network modeling results indicated that speed of information processing and task-related demands significantly influenced P3AERP latency in children with auditory processing disorders. Competing noise and rapid stimulus rates influenced P3AERP amplitude in both groups.

\section{Introduction}

Auditory processing is the ability of the central auditory nervous system (CANS) to use and process auditory information received peripherally by the two ears. Auditory processing disorders (APD) are typically seen in individuals with normal hearing sensitivity and are characterized by an inability of the central auditory neurons to mediate higher-order auditory processing skills (e.g., speech in noise, binaural processing, temporal processing, and closure). Individuals with APD manifest listening difficulties in challenging listening conditions, show deficits in spatial location (localization) of sounds, and face difficulties in decoding rapid rate stimuli [1]. The effects of APD can be devastating because as an input disorder, it has the potential to impair the abilities for spoken language comprehension, learning, and cognition in schoolage children.

One of the main problems in identification of APD is that this disorder often coexists with other comorbid conditions in school-age children such as attention deficit disorders, language learning disorders, and learning disabilities [2]. This makes differential diagnosis of APD difficult. Also audiologists routinely use primarily language-based auditory processing measures for diagnosis of APD even though it is not clear whether deficits on linguistic (verbal) tasks are more likely to be associated with APD than nonlinguistic (e.g., tonal) tasks. In a study by Rosen et al. [3], it has been shown that school-age children with suspected APD exhibited poorer performance on auditory tests in both verbal (Consonant Cluster Minimal Pairs) and tonal (Tallal Discrimination Task) conditions, relative to age-matched controls. There is also dispute regarding formulation of the appropriate test battery for evaluation of APD (e.g., $[4,5])$. Cacace and McFarland $[4,5]$ contend that, for a diagnosis of APD, testing should address the primary deficit in processing of acoustic information in the auditory modality and deficits should be shown to be absent or reduced in other (e.g., visual) modalities. While this notion is disputed by other studies $[6,7]$, there is consensus on the need for valid tools that challenge listening in the auditory modality for school-age children with APD.

P300 Auditory Event-Related Potentials (P3AERPs) have received increasing attention in the assessment of APD [811]. P3AERPs are scalp-recorded positive potentials with a latency approximating $300 \mathrm{msec}$ from stimulus onset and are widely recognized as physiological measures of cognitive processing [12-14]. P3AERPs are acquired using an oddball paradigm. Subject responses to frequent stimuli (ignored by listener) are averaged separately from responses to the rare or infrequent stimuli (attended to by listener). P3AERPs are typically considered to be endogenous potentials that are influenced more by internal (subject-related) factors than 
external (stimulus-related) factors $[8,15]$. However, several studies have also shown that stimulus-related factors (e.g., frequency and intensity) can significantly influence latency and amplitude of P3AERPs [16-19].

P3AERPs provide a good index of brain activity related to the mental representation of incoming stimuli [20]. Initially, sensory processing occurs and is followed by an attention-drive comparison process that evaluates the initial sensory impression with a change in stimulus (novelty on mismatch) and results in cortex-updating P300 generation [21]. P3AERPs appear to have promise in evaluation of the functional status of the CANS and can add valuable information to behavioral tests currently in use for evaluation of APD. The P3AERP represents a positive potential thought to be generated from the frontal lobe, polarity event-related $[22,23]$. The P3AERP reflects a response that is based on task relevance assigned to a specific stimulus [24]. However, there is currently limited clinical use of P3AERPs in the evaluation of APD, primarily due to factors such as high cost, lack of training, and need for specialized software. A recent survey showed that only 14/130 (11\%) of participating clinicians used cortical event-related potentials as part of their test battery [25]. In this survey, respondents disagreed with use of a minimum test battery proposed at the Bruton Conference Statement [2].

Latency of P3AERPs is believed to index stimulus classification speed and is proportional for the time taken to detect and evaluate a larger novel stimulus in the context of other frequently prescribed stimuli [21]. Latency of P3AERPs can be a useful measure of the speed of information processing in the central auditory nervous system (CANS); that is, the faster the information processing, the shorter the P3AERP latency $[13,14]$. The typical latency of the scalp-recorded vertex-positive P3AERPs in normal listeners is approximately 300 milliseconds ( $\mathrm{msec}$ ) from stimulus onset at slow stimulus rates $[13,26]$. Longer P3AERP latencies have been reported in individuals with lesions and disorders of the CANS [10, 11]. Jirsa and Clontz [27] investigated P3AERPs in children and found that children with APD showed longer P3AERP latencies than age-matched controls without APD. P3AERP latencies were compared in adults with APD and control adults (without APD) in binaural and competing noise conditions [28]. Adults with APD showed significantly longer P3AERP latencies than control adults without APD in competing noise conditions where competing noise was presented along with the frequent and infrequent stimuli on the P3AERP task [28]. Hence, P3AERPs appear to have promise in evaluation of the functional status of the CANS and can add considerable value in the assessment of APD.

P3AERP amplitude measures can provide indices of the amount of neurological substrate available for information processing [29]. The typical amplitude of P3AERPs in normal listeners is approximately $12-15 \mu \mathrm{V}[13,26]$. P3AERP amplitude is inversely related to stimulus probability: the less frequently a stimulus is presented, the larger the amplitude is and vice versa $[30,31]$. P3AERP amplitudes were found to be significantly larger in control subjects (with no CANS lesions) than in patients with known CANS lesions [29]. Significantly larger P3AERP amplitudes were also reported in children without APD than in children with APD in an investigation by Jirsa and Clontz [27].

The effects of less favorable listening conditions (e.g., rapid rates and competing noise) on P3AERP latency and amplitude have received limited attention in individuals with APD. Many of the P3AERP studies in children have been typically conducted under favorable (e.g., binaural tone bursts and no competing noise) listening conditions (e.g., [27, 32]). However, current behavioral tests in clinical use for APD are designed on the basis that children with APD will typically show a breakdown in auditory processing only under adverse listening conditions where extrinsic redundancy is reduced, such as spectral filtering, competing noise, or rapid stimuli [33]. Hence, there is a strong need to study P3AERPs under similar adverse listening situations in children with and without APD prior to clinical use of P3AERPs as a tool for evaluation of APD [34].

Neural networks are adaptive statistical models based on analogies with human brain structure that can learn to estimate and iteratively change values of the parameters of some population using specific input and output variables [35]. An artificial neural network (ANN), often just called a "neural network" (NN), is a mathematical model or computational model based on biological neural networks. Artificial neural networks can be used to model complex relationships between input and output variables and explain patterns of data. The construction of the neural network typically involves three different layers with feed-forward architecture. This is the most popular network architecture in use today. The input layer of this network is a set of input units, neurons that are fully connected to the hidden layer with the hidden units that are in turn fully connected to an output layer. The output layer supplies the response of neural network to the activation pattern applied to the input layer. Neural network modeling has been used in healthcare research to characterize and predict a wide variety of health-related issues such as infant mortality [36], brain surgery decisions [37], pharmacokinetic parameters of antibiotics in severely ill patients [38], and auditory dysfunction in Alzheimer's disease [39].

Neural networks can be used to model cognitive processes by a feed-forward, backward propagation algorithm called multilayer perceptrons (MLPs). These networks usually organize their units into several layers. The information to be analyzed is fed to the first layer called the input layer, followed by intermediate hidden layers, finally leading to the output layer for processing [35]. Unlike multiple linear regression models used to predict performance from known variables, artificial neural networks need no prior knowledge or assumptions because they can learn and generalize from data that are even noisy or imperfect [40].

The current study was conducted to probe if reducing extrinsic redundancy in the P3AERP task compromises auditory processing in school-age children with and without APD. Extrinsic redundancy can be reduced in several ways, but, for the purposes of this study, two stimulus-related variables (competing noise and rapid rates) were used. The rationale for reducing the extrinsic redundancy was that competing noise would limit spectral processing abilities needed to 
TABLE 1: Screening results for children with APD.

\begin{tabular}{|c|c|c|c|c|c|c|c|c|c|}
\hline \multirow{2}{*}{$\#$} & \multirow{2}{*}{ Age } & \multirow{2}{*}{ Fisher's checklist } & \multicolumn{4}{|c|}{ SCAN } & \multicolumn{3}{|c|}{ ABR latency (msec) } \\
\hline & & & FW & AFG & $\mathrm{CW}$ & Composite & I & III & $\mathrm{V}$ \\
\hline 1 & 9 y $9 \mathrm{~m}$ & $48 \%{ }^{* *}$ & $3^{*}$ & 8 & 5 & $69^{*}$ & 1.5 & 3.5 & 5.5 \\
\hline 2 & 8 y $2 \mathrm{~m}$ & $16 \%^{* *}$ & 14 & 6 & $4^{*}$ & $69^{*}$ & 1.7 & 3.7 & 5.3 \\
\hline 3 & 8 y $5 \mathrm{~m}$ & $36 \%{ }^{* *}$ & 6 & 7 & 5 & $68^{*}$ & 1.6 & 3.6 & 5.6 \\
\hline 4 & 9 y $5 \mathrm{~m}$ & $36 \%^{* *}$ & 14 & 6 & $4^{*}$ & $69^{*}$ & 1.6 & 3.8 & 5.6 \\
\hline 5 & 12 y $2 \mathrm{~m}$ & $40 \%{ }^{* *}$ & $1^{*}$ & $4^{*}$ & $1^{*}$ & $37^{*}$ & 1.7 & 3.7 & 5.7 \\
\hline 6 & $13 y$ & $48 \%{ }^{* *}$ & $1^{*}$ & $2^{*}$ & $1^{*}$ & $33^{*}$ & 1.8 & 3.8 & 5.5 \\
\hline 7 & 12 y $2 \mathrm{~m}$ & $48 \%{ }^{* *}$ & 6 & 6 & 6 & $65^{*}$ & 1.7 & 3.4 & 5.7 \\
\hline 8 & 11 y $7 \mathrm{~m}$ & $36 \%{ }^{* *}$ & 7 & 7 & $3^{*}$ & $62^{*}$ & 1.6 & 3.5 & 5.6 \\
\hline 9 & 12 y $9 \mathrm{~m}$ & $48 \%{ }^{* *}$ & $3^{*}$ & $4^{*}$ & 5 & $58^{*}$ & 1.7 & 3.7 & 5.7 \\
\hline
\end{tabular}

* 2 SD below mean norms.

${ }^{* *}$ Below age norms or grade norms.

TABLE 2: Screening results for children without APD.

\begin{tabular}{|c|c|c|c|c|c|c|c|c|c|}
\hline \multirow{2}{*}{ \# } & \multirow{2}{*}{ Age } & \multirow{2}{*}{ Fisher's checklist } & \multicolumn{4}{|c|}{ SCAN } & \multicolumn{3}{|c|}{ ABR latency (msec) } \\
\hline & & & FW & AFG & CW & Composite & I & III & $\mathrm{V}$ \\
\hline 1 & 9 y $5 \mathrm{~m}$ & $80 \%$ & 9 & 7 & 9 & 88 & 1.5 & 3.5 & 5.5 \\
\hline 2 & $8 y$ & $88 \%$ & 7 & 8 & 6 & 81 & 1.7 & 3.7 & 5.3 \\
\hline 3 & 8 y $5 \mathrm{~m}$ & $76 \%$ & 9 & 9 & 8 & 92 & 1.6 & 3.6 & 5.6 \\
\hline 4 & 9 y $5 \mathrm{~m}$ & $76 \%$ & 12 & 9 & 8 & 97 & 1.6 & 3.8 & 5.6 \\
\hline 5 & 12 y $1 \mathrm{~m}$ & $72 \%$ & 11 & 8 & 8 & 77 & 1.7 & 3.7 & 5.7 \\
\hline 6 & $13 y$ & $72 \%$ & 11 & 9 & 7 & 98 & 1.8 & 3.8 & 5.5 \\
\hline 7 & 11 y $7 \mathrm{~m}$ & $96 \%$ & 14 & 8 & 6 & 84 & 1.7 & 3.4 & 5.7 \\
\hline 8 & $12 \mathrm{y}$ & $76 \%$ & 7 & 10 & 9 & 81 & 1.6 & 3.5 & 5.6 \\
\hline 9 & 12 y $10 \mathrm{~m}$ & $96 \%$ & 13 & 8 & 11 & 106 & 1.7 & 3.7 & 5.7 \\
\hline
\end{tabular}

discriminate frequent and infrequent stimuli on the P3AERP task while rapid presentation rates would stress the temporal processing capabilities of the auditory system and these would have particular influence on P3AERP latency and amplitude measures in those children with reduced intrinsic redundancy (children with APD). Neural network modeling was performed statistically to discover hidden and nonlinear associations between input (stimulus rate and competing noise) and output variables (P3AERP latency and amplitude).

\section{Methods}

2.1. Subjects. A total of eighteen subjects were categorized into two groups: (1) 9 children with APD (mean age: 10 years and 9 months; age range: 8 years and 5 months to 13 years) and (2) 9 age-matched and gender-matched children without APD (mean age: 10 years and 9 months; age range: 8 years to 13 years). Age matching for children with and without APD was done to ensure a difference not exceeding 6 months between matched subjects. Children with APD were selected from the patient files of the Auburn University Speech and Hearing Clinic. Age- and gender-matched children without APD were recruited from the local school system. Parents of all subjects had to sign informed consent in accordance with Institutional Review Board Guidelines prior to participation. Each subject received a \$30 payment towards travel and participation in the study.

Screening protocols were completed first for all participating subjects in the study. Otoscopy was followed by a complete audiological evaluation in both ears performed on a two-channel Madsen OB 822 audiometer [41]. In order to be included in the study, subjects from both groups had to show normal hearing sensitivity (hearing threshold $<25 \mathrm{~dB}$ at frequencies $500 \mathrm{~Hz}-8000 \mathrm{~Hz}$ ). All subjects were tested in an audiometric booth within the Auditory Research Lab in the Auburn University Speech and Hearing Clinic with ambient noise below recommended levels [42]. Fisher's auditory checklist [43] was used to screen for poor listening skills (below grade norms) in school-age children (see results in Tables 1 and 2). Children who exhibited severe language and/or reading problems during the Fisher's checklist completion were excluded to reduce possible comorbid effects. Subjects were then screened to ensure normal middle ear function (Jerger type "A" tympanograms bilaterally) on a Madsen ZO 33 immittance meter. Normal brainstem function was ensured on the ABR test (see latencies in Tables 1 and 2) prior to participation in the P3AERP experimental protocol for all subjects.

All subjects were first screened for central auditory function (see results in Tables 1 and 2) using the SCAN or 
TABLE 3: Behavioral test results for children with APD.

\begin{tabular}{|c|c|c|c|c|c|c|c|}
\hline \multirow{2}{*}{ \# } & \multirow{2}{*}{ Age } & \multicolumn{3}{|c|}{ PPT } & \multicolumn{3}{|c|}{ DPT } \\
\hline & & Norms $^{*}$ & Right ear & Left ear & Norms $^{*}$ & Right ear & Left ear \\
\hline 1 & 9 y $9 \mathrm{~m}$ & $>/=65 \%$ & $48 \%{ }^{* *}$ & $62 \%^{* *}$ & $>/=65 \%$ & $27 \%$ & $32 \%^{* *}$ \\
\hline 2 & 8 y 2 m & $>/=40 \%$ & $38 \%{ }^{* *}$ & $21 \%{ }^{* *}$ & $>/=40 \%$ & $28 \%^{* *}$ & $30 \%{ }^{* *}$ \\
\hline 3 & 8 y $5 \mathrm{~m}$ & $>/=40 \%$ & $37 \%{ }^{* *}$ & $42 \%$ & $>/=40 \%$ & $38 \%{ }^{* *}$ & $38 \%{ }^{* *}$ \\
\hline 4 & 9 y $5 \mathrm{~m}$ & $>/=65 \%$ & $85 \%$ & $62 \%^{* *}$ & $>/=65 \%$ & $60 \%{ }^{* *}$ & $73 \%$ \\
\hline 5 & 12 y $2 \mathrm{~m}$ & $>/=75 \%$ & $50 \%{ }^{* *}$ & $48 \%{ }^{* *}$ & $>/=75 \%$ & $76 \%$ & $44 \%^{* *}$ \\
\hline 6 & $13 \mathrm{y}$ & $>/=75 \%$ & $84 \%$ & $60 \%{ }^{* *}$ & $>/=75 \%$ & $56 \%{ }^{* *}$ & $52 \%{ }^{* *}$ \\
\hline 7 & 12 y $2 \mathrm{~m}$ & $>/=75 \%$ & $52 \%{ }^{* *}$ & $56 \%{ }^{*}$ & $>/=75 \%$ & $44 \%^{* *}$ & $24 \%^{* *}$ \\
\hline 8 & 11 y $7 \mathrm{~m}$ & $>/=75 \%$ & $70 \%{ }^{* *}$ & $78 \%$ & $>/=75 \%$ & $78 \%$ & $60 \%{ }^{* *}$ \\
\hline 9 & 12 y $9 \mathrm{~m}$ & $>/=75 \%$ & $72 \%{ }^{* *}$ & $64 \%^{* *}$ & $>/=75 \%$ & $36 \%^{* *}$ & $36 \%{ }^{* *}$ \\
\hline
\end{tabular}

${ }^{*}$ From Bellis [52].

** Below norms.

TABLE 4: Behavioral test results for children without APD.

\begin{tabular}{|c|c|c|c|c|c|c|c|}
\hline \multirow{2}{*}{$\#$} & \multirow{2}{*}{ Age } & \multicolumn{3}{|c|}{ PPT } & \multicolumn{3}{|c|}{ DPT } \\
\hline & & Norms ${ }^{*}$ & Right ear & Left ear & Norms ${ }^{*}$ & Right ear & Left ear \\
\hline 1 & 9 y $5 \mathrm{~m}$ & $>/=65 \%$ & $75 \%$ & $68 \%$ & $>/=65 \%$ & $84 \%$ & $90 \%$ \\
\hline 2 & $8 y$ & $>/=40 \%$ & $68 \%$ & $72 \%$ & $>/=40 \%$ & $40 \%$ & $45 \%$ \\
\hline 3 & 8 y $5 \mathrm{~m}$ & $>/=40 \%$ & $98 \%$ & $90 \%$ & $>/=40 \%$ & $57 \%$ & $48 \%$ \\
\hline 4 & 9 y $5 \mathrm{~m}$ & $>/=65 \%$ & $75 \%$ & $80 \%$ & $>/=65 \%$ & $65 \%$ & $65 \%$ \\
\hline 5 & 12 y $1 \mathrm{~m}$ & $>/=75 \%$ & $95 \%$ & $92 \%$ & $>/=75 \%$ & $81 \%$ & $83 \%$ \\
\hline 6 & $13 y$ & $>/=75 \%$ & $85 \%$ & $85 \%$ & $>/=75 \%$ & $78 \%$ & $78 \%$ \\
\hline 7 & 11 y 7 m & $>/=75 \%$ & $78 \%$ & $78 \%$ & $>/=75 \%$ & $80 \%$ & $80 \%$ \\
\hline 8 & $12 y$ & $>/=75 \%$ & $80 \%$ & $80 \%$ & $>/=75 \%$ & $76 \%$ & $78 \%$ \\
\hline 9 & 12 y $10 \mathrm{~m}$ & $>/=75 \%$ & $100 \%$ & $90 \%$ & $>/=75 \%$ & $100 \%$ & $90 \%$ \\
\hline
\end{tabular}

${ }^{*}$ From Bellis [52].

SCAN-A test battery $[44,45]$. The subtests in the SCAN screening battery for children included (a) Filtered Words (to evaluate auditory closure), (b) Auditory Figure-Ground (to evaluate speech in noise), and (c) Competing Words (to evaluate dichotic speech). Screening by these tests provides consideration of the following factors: (a) monaural separation/closure, (b) binaural integration, and (c) binaural separation $[46,47]$.

The two auditory pattern perception tests used for clinical assessment of APD were (1) Pitch Patterns Test (PPT) [48] and (2) Duration Patterns Test (DPT) [49]. Both of these measures are highly sensitive to lesions in the CANS $[48,50$, 51] and performance of children with APD can be compared to age norms available for school-age children on both of these tests $[10,52]$. These tests were also selected because they can provide useful measures of auditory pattern recognition and temporal ordering [46, 47]. Tables 3 and 4 show the performance of all subjects in behavioral tests.

2.2. Recording Procedures. A four-channel electrode montage was used to record P3AERPs on a Cadwell Excel Evoked Potentials System. In accordance with the International 10-20 System, neuroelectrical activity was recorded from silver cup electrodes placed on the midline at frontal $\left(F_{z}\right)$, central $\left(C_{z}\right)$, and parietal $\left(P_{z}\right)$ scalp locations, referenced to linked electrodes on earlobes and a forehead ground
$\left(F_{p z}\right)$. Myogenic activity from eye movement was monitored by electrooculographic (EOG) recording from electrodes placed below and above the left eye. Trials contaminated by eye movements or myogenic artifacts were automatically excluded from the averages. It was ensured that all individual electrode impedances were below 3000 ohms and the interelectrode impedances were below $1000 \mathrm{ohms}$.

Subjects were seated in a reclining chair and required to fixate their vision on a target placed in front of them to minimize visual or movement artifacts. All subjects were instructed and trained to listen for the rare or infrequent high-pitched $(2000 \mathrm{~Hz})$ tones and ignore the lower pitched frequent $(1000 \mathrm{~Hz})$ tones. All subjects were required to keep count of the number of infrequent stimuli for report to the investigator after the respective trial. This ensured selective attention required for the P3AERP task. Practice trials were provided for all subjects prior to the experiment. The entire procedure took approximately two hours for each subject and rest was provided between some experimental conditions in children to reduce fatigue.

2.3. Stimuli. Rarefaction tone bursts with intensity of $70 \mathrm{~dB}$, duration of 20 milliseconds (msec), and rise-fall time of $10 \mathrm{msec}$ were used. An oddball paradigm was selected to assess auditory discrimination of the frequent and infrequent stimuli. The frequent stimuli $(1000 \mathrm{~Hz}$ tone bursts) and 
infrequent stimuli (2000 Hz tone bursts) were presented binaurally in a $4: 1$ (frequent : infrequent) ratio in an oddball paradigm through insert ER3 phones at rates of 1/sec and $3 /$ sec. Stimuli (frequent and infrequent tone bursts) were presented along with competing noise presented in both ears. The stimuli were presented at $70 \mathrm{~dB}$ HL while the level of competing noise was varied $(0 \mathrm{~dB}, 40 \mathrm{~dB}$, and $60 \mathrm{~dB})$.

2.4. Conditions. Each subject was evaluated in six P3AERP conditions: (1) $1 / \mathrm{sec}$ rate, $0 \mathrm{~dB}$ noise, (2) $1 / \mathrm{sec}$ rate, $40 \mathrm{~dB}$ noise, (3) $1 / \mathrm{sec}$ rate, $60 \mathrm{~dB}$ noise, (4) $3 / \mathrm{sec}$ rate, $0 \mathrm{~dB}$ noise, (5) $3 / \mathrm{sec}, 40 \mathrm{~dB}$ noise, and (6) $3 / \mathrm{sec}, 60 \mathrm{~dB}$ noise. The order of conditions was counterbalanced across subjects to reduce any order effects.

2.5. Data Analysis. P3AERPs were identified and latencies and amplitudes were determined for P3AERP waves identified on the basis of a comparison between the frequent and infrequent waveforms. Two runs of each condition were required for repeatability and reliability purposes. For singlepeaked replicable waveforms, the P3AERP component was identified as the large positive peak following the N200 component and present between 250 and $700 \mathrm{~ms}$ in the infrequent waveform but absent or of reduced amplitude in the frequent waveform $[32,53]$. The N200 component was defined as the largest negative trough following P200 in a latency range between 150 and $250 \mathrm{~ms}$. For multiple-peaked or broad-peaked waveforms, an intersect method was used $[53,54]$. Intersection of extrapolated lines from the ascending and descending slopes of the multiple-peaked or broadpeaked positive P3AERP components following N200 in the infrequent waveform was used to determine the P3AERP component.

Latencies were measured to the highest peak of the P3AERP wave for single-peaked waveforms described above or by the slope-intersect for multiple-peaked waveforms described above. Amplitudes were measured from the N200 trough to the P3AERP peak (for single-peaked waveforms) or from the N200 trough to the slope-intersect (for multiplepeaked waveforms). For waveforms to be accepted for analysis and interpretation, identification of the P3AERP peak by at least two of three independent experienced raters with good confidence ratings of 3 or higher on a 5-point rating scale was required [55].

There was excellent agreement between recordings obtained from the frontal $\left(F_{z}\right)$, central $\left(C_{z}\right)$, and parietal $\left(P_{z}\right)$ scalp locations across all subjects $(r=0.96)$. Data recorded from central $\left(C_{z}\right)$ locations were used for analysis because $C_{z}$ scalp locations provide good topography for amplitude/latency correlations that reflect neurocognitive operations underlying fundamental discrimination processes required in the P3AERP oddball paradigm [56]. P3AERP latency and amplitude measures were averaged over two complete trials and the data were subjected to factorial analyses of variance to investigate effects of groups (APD versus non-APD), stimulus rate $(1 / \mathrm{sec}$ versus $3 / \mathrm{sec}$ ), and competing noise (no noise versus $40 \mathrm{~dB}$ noise versus $60 \mathrm{~dB}$ noise). Figures 1 and 2 show typical P3AERP recordings from $C_{z}, P_{z}$, and $F_{z}$ locations along with electrooculographic

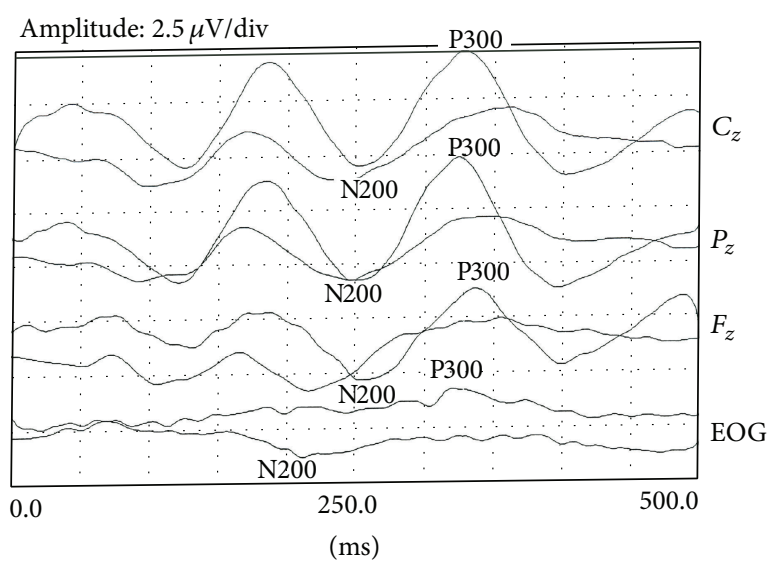

Latency: $50 \mathrm{~ms} / \mathrm{div}$

Figure 1: Representative P300 waveforms for a child with APD.

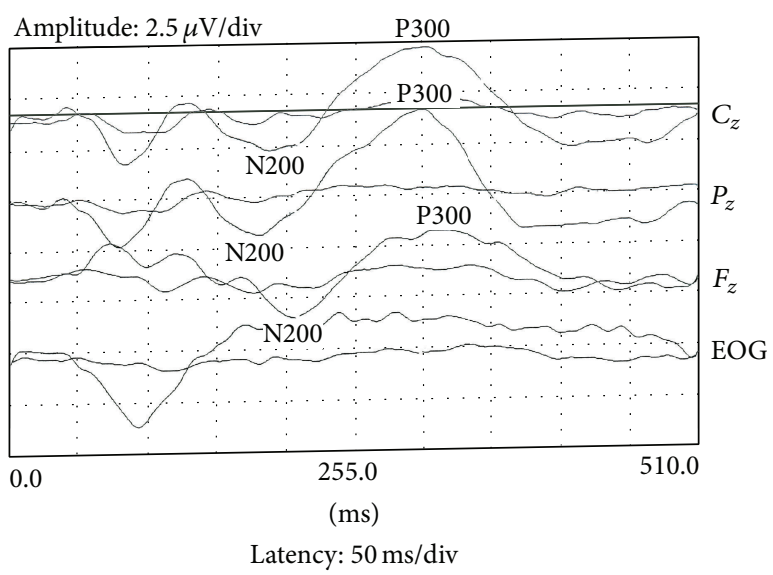

FIgURE 2: Representative P300 waveforms for a child without APD.

(EOG) recordings in a child with APD and a control child, respectively. Please note that in Figures 1 and 2, the higheramplitude wave marked "P300" was found for recordings associated with the infrequent stimuli in all electrode $\left(C_{z}, P_{z}\right.$, and $F_{z}$ ) locations.

2.6. Neural Network Modeling. P3AERP latency and amplitude data were subjected to neural network algorithms to find possible hidden associations between input (group- and stimulus-related) and output (P3AERP) variables. The multilayer perceptron architecture used in the analysis is popular to approximate any multivariate relationship between input and output variables [35]. A hidden layer collects information from the units of the input layer and looks for weighted connections called synapses. These synaptic weights influence (enhance or decrease) the input information to produce the resultant outcome seen in the output layer. A positive synaptic weight $(>0)$ is considered excitatory while a negative weight $(<0)$ is considered inhibitory. In our study, the input layer of the neural network analysis included stimulus parameters and output patterns included P3AERP latency and amplitude. 


\subsubsection{Neural Network Modeling}

(1) Software. All the neural network modeling was completed in IBM SPSS Version 20 software. In IBM SPSS Modeler, the neural networks used are feed-forward neural networks, also known as multilayer perceptrons. The neurons in such networks (sometimes called units) are arranged in layers. Typically, there is one layer for input neurons, one or more internal processing hidden layers, and one output layer. Each layer is connected to every neuron in the hidden layer, and each neuron in the hidden layer is connected to every neuron in the output layer.

The connections between neurons have weights associated with them, which determine the strength of influence one neuron has on another. Information flows from the input layer via the processing layer to the output layer to generate predictions. By adjusting the connection weights during training to match predictions, the network "learns" to generate better and better predictions.

The training of a multilayer perceptron uses a method called back propagation of error, based on the generalized delta rule [57]. For each record presented to the network to generate a prediction from the output layer, this prediction is compared to the recorded output value for the training record, and the difference between the predicted and actual output(s) is propagated backward through the network to adjust the connection weights to improve the prediction for similar patterns.

(2) Architecture. The IBM SPSS Modeler uses the multilayer perceptron (MLP), a feed-forward, supervised learning network with up to two hidden layers. The MLP network is a function of one or more predictors which minimizes the prediction error of one or more targets. The general architecture for the MLP modeling consists of an input layer, hidden layer, and output layer. Expert architecture selection determines the "best" number of hidden units in a single hidden layer. All of the data set is used if the number of records is less than 1000. A random sample is taken from the entire data set and split into training (70\%) and testing samples (30\%). Error back propagation is used to compute the error function and adjust synaptic weights of the variables.

(3) Supervised Learning Rule. In neural networks, learning rules are provided with a set of input-output data (also called training data) of proper network behavior. As the inputs are applied to the network, the network outputs are compared to the target outputs. The learning rule is then used to adjust the weights and biases of the network in order to move the network outputs closer to the targets. The Widrow-Hoff learning rule [58] is widely used for supervised training of neural networks. It is independent of the activation function of the neurons used since it minimizes the squared error between the desired output and neuron's activation value. This rule can be considered a special case of delta learning rule. For training purposes, the Widrow-Hoff rule was applied to use differences between actual inputs and desired outputs as the error signal for the estimation of units in the output layer. The model performance was cross-validated by assigning $70 \%$ of cases for training and $30 \%$ of cases for testing.

(4) Feed-Forward, Back-Propagation Algorithm. The feedforward back-propagation learning algorithm is a wellrecognized procedure for training neural networks for multilayer perceptrons (MLPs). It is based on plotting performance error as a function of neural network weights. Each iteration in the algorithm constitutes two sweeps: forward activation to produce a solution and a backward propagation of the computed error to modify the weights. The back-propagation algorithm [57] is used in layered feed-forward ANNs. This means that the artificial neurons are organized in layers and send their signals "forward," and then the errors are propagated backwards. The network receives inputs by neurons in the input layer, and the output of the network is given by the neurons on an output layer. There may be one or more intermediate hidden layers. The back-propagation algorithm uses supervised learning, which means that we provide the algorithm with examples of the inputs and outputs we want the network to compute, and then the error (difference between actual and expected results) is calculated. The idea of the back-propagation algorithm is to reduce this error, until the ANN learns the training data. The training begins with random weights, and the network begins to adjust them so that the error will be minimal. The neural network paradigm used in this study utilized the back-propagation neural networks with a single hidden layer that have been shown to be capable of providing an accurate approximation of any continuous function provided that there are sufficient hidden neurons.

(5) Hidden Layers. The SPSS algorithm used a single hidden layer in our study because it has been shown that an MLP with one hidden layer has the capacity to approximate any function with an acceptable degree of accuracy if there are enough hidden nodes.

\section{Results}

3.1. Response Reliability. There were no significant $(P>0.05)$ differences in the numbers of infrequent stimuli counted between the two groups of children (with and without APD). Intragroup comparisons showed that all individual subjects were within $\pm 10 \%$ of the target count. Interjudge reliability on P3AERP analyses across judges was good $(r=0.90)$. P3AERPs were obtained in six conditions: (1) $1 / \mathrm{sec}$ rate, $0 \mathrm{~dB}$ noise, (2) $1 / \mathrm{sec}$ rate, $40 \mathrm{~dB}$ noise, (3) $1 / \mathrm{sec}$ rate, $60 \mathrm{~dB}$ noise, (4) $3 / \mathrm{sec}$ rate, $0 \mathrm{~dB}$ noise, (5) 3/sec, $40 \mathrm{~dB}$ noise, and (6) 3/sec, $60 \mathrm{~dB}$ noise.

\subsection{Analyses of Variance Results}

3.2.1. P3AERP Latencies. Analyses of variance (ANOVA) results (see Table 5) showed significant differences in P3AERP latency between the groups of children with and without APD $(F(1,96)=13.55 ; P<0.01)$. Post hoc (Fisher's LSD) means comparisons showed significantly $(P<0.01)$ greater mean latencies for children with APD (344.08 msec) 
TABLE 5: ANOVA results for P300 latencies.

\begin{tabular}{|c|c|c|c|c|c|}
\hline Effect & $\mathrm{dF}$ & MS effect & MS error & $F$ & $P$ \\
\hline Groups & 1 & 9357.66 & 690.30 & 13.55 & $<0.01^{*}$ \\
\hline Rate & 1 & 9383.75 & 690.30 & 13.59 & $<0.01^{*}$ \\
\hline Noise & 2 & 440.48 & 690.30 & 0.64 & 0.53 \\
\hline Groups $\times$ rate & 1 & 22.87 & 690.30 & 0.03 & 0.86 \\
\hline Groups $\times$ noise & 2 & 937.95 & 690.30 & 1.36 & 0.26 \\
\hline Rate $\times$ noise & 2 & 1053.88 & 690.30 & 1.52 & 0.22 \\
\hline Groups $\times$ rate $\times$ noise & 2 & 509.34 & 690.30 & 0.74 & 0.48 \\
\hline
\end{tabular}

${ }^{*}$ Statistically significant difference $(P<0.05)$.

TABLE 6: ANOVA results for P300 amplitudes.

\begin{tabular}{lccccc}
\hline Effect & dF & MS effect & MS error & $F$ & 2.45 \\
\hline Groups & 1 & 30.04 & 12.28 & 12.28 & 20.39 \\
Rate & 1 & 250.49 & 12.28 & 0.96 & 0.12 \\
Noise & 2 & 11.91 & 12.28 & 0.89 & 0.38 \\
Groups $\times$ rate & 1 & 10.94 & 12.28 & 1.93 & 0.35 \\
Groups $\times$ noise & 2 & 23.74 & 12.28 & 1.48 & 0.15 \\
Rate $\times$ noise & 2 & 18.27 & 12.28 & 1.37 & 0.23 \\
Groups $\times$ rate $\times$ noise & 2 & 16.81 & & 0.26 \\
\hline
\end{tabular}

${ }^{*}$ Statistically significant difference $(P<0.05)$.

TABLE 7: Descriptive statistics for P300 latencies.

\begin{tabular}{|c|c|c|c|c|c|c|}
\hline & \multicolumn{3}{|c|}{ Rate $=1 / \mathrm{sec}$} & \multicolumn{3}{|c|}{ Rate $=3 / \mathrm{sec}$} \\
\hline & No noise & $30 \mathrm{~dB}$ noise & $60 \mathrm{~dB}$ noise & No noise & $30 \mathrm{~dB}$ noise & $60 \mathrm{~dB}$ noise \\
\hline APD group & $324.22(18.14)$ & $334.42(17.76)$ & $344.28(15.84)$ & $345.5(29.95)$ & $356.78(37.18)$ & $359.33(35.72)$ \\
\hline Non-APD group & 310.05 (18.48) & 314.55 (22.22) & $325.22(20.22)$ & $344.94(28.14)$ & $333.22(33.03)$ & $323.83(26.54)$ \\
\hline
\end{tabular}

TABLE 8: Descriptive statistics for P300 amplitudes.

\begin{tabular}{|c|c|c|c|c|c|c|}
\hline & \multicolumn{3}{|c|}{ Rate $=1 / \mathrm{sec}$} & \multicolumn{3}{|c|}{ Rate $=3 / \mathrm{sec}$} \\
\hline & No noise & $30 \mathrm{~dB}$ noise & $60 \mathrm{~dB}$ noise & No noise & $30 \mathrm{~dB}$ noise & $60 \mathrm{~dB}$ noise \\
\hline APD group & $\begin{aligned} \mathrm{M} & =8.23 \\
\mathrm{SD} & =4.05\end{aligned}$ & $7.45(3.96)$ & $5.89(3.39)$ & $4.10(2.76)$ & 4.69 (1.95) & $5.54(2.88)$ \\
\hline Non-APD group & $8.77(3.18)$ & $7.02(3.17)$ & $10.85(5.35)$ & $3.53(1.26)$ & $5.64(3.46)$ & $6.42(3.62)$ \\
\hline
\end{tabular}

than mean latencies of children without APD (325.47 msec), indicating differences in speeds of information processing between children with and without APD. Main effects of stimulus rate were also significant $(F(1,96)=13.59$; $P<0.01)$ and post hoc means comparisons using Fisher's LSD indicated significantly longer mean latencies (344.11 msec) for the $3 / \mathrm{sec}$ condition than mean latencies for the $1 / \mathrm{sec}$ condition $(325.45 \mathrm{msec}$ ), indicating that increasing stimulus rate increases P3AERP latency. The main effects of competing noise were not significant $(F(2,96)=0.63$; $P>0.05)$. Descriptive statistics of P3AERP latencies are shown in Table 7.

3.2.2. P3AERP Amplitudes. Analyses of variance (ANOVA) results (see Table 6) showed no significant $(P>0.05)$ differences in P3AERP amplitude between the groups of children with and without $\operatorname{APD}(F(1,96)=2.45 ; P=0.12)$. Main effects of stimulus rate were significant $(F(1,96)=$ 20.39; $P<0.01)$. Post hoc means comparisons using Fisher's least significant difference (LSD) showed significantly $(P<0.01)$ greater mean amplitudes $(8.03 \mu \mathrm{V})$ for the $1 / \mathrm{sec}$ condition than for the $3 / \mathrm{sec}$ condition $(4.99 \mu \mathrm{V})$. Main effects of competing noise were not significant $(F(2,96)=0.97$; $P>0.05)$. Descriptive statistics of P3AERP amplitudes are shown in Table 8.

\subsection{Neural Network Modeling}

3.3.1. Context Updating Model [21]. Polich [21] has provided an updating theory for P300 generation and has proposed three factors: (1) processing capacity, (2) attention allocation, and (3) task demands. According to Polich [21], the initial 


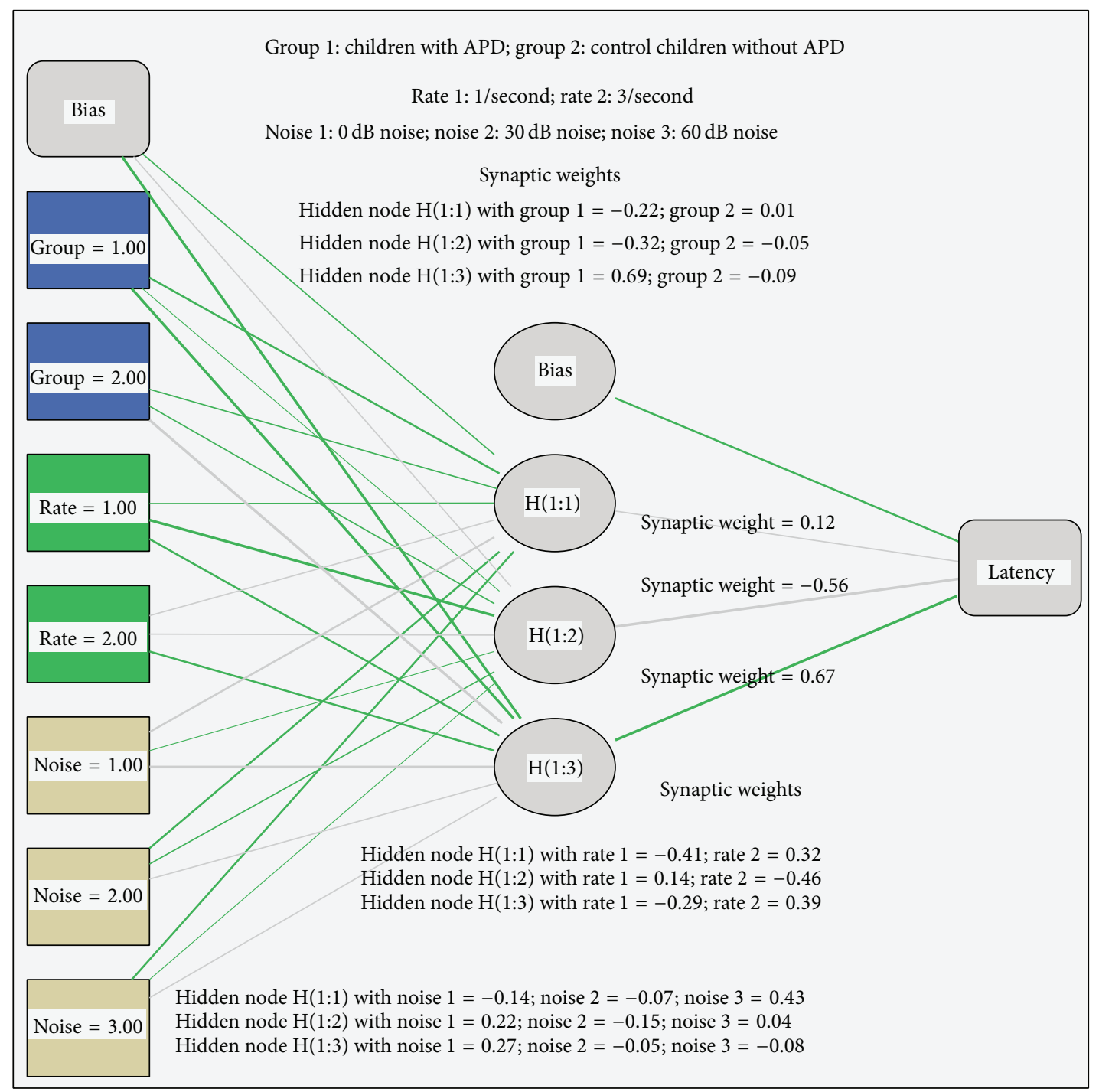

Figure 3

sensory processing for the P300 task is fundamental for stimulus classification and the processing capacity factor can limit this processing. The second factor, "attention allocation," reflects an attention driven process that evaluates stimulus classification (target or novel versus frequent), by evaluating the comparison in working memory. P3AERP latency and amplitude are strongly influenced by this attention allocation factor and active attention increases P3AERP amplitude while decreasing P3AERP latency. The third factor, "task demands," postulates that task requiring greater amounts of attentional resources will increase P3AERP amplitude and/or increase P3AERP latency.

3.4. Latency Results for P3AERP. The results of neural network analyses for P3AERP latency results are shown in Figure 3. For the three factors (groups, rate, and noise), there were a total of seven units comprised of two units for groups, two units for rate, and three units for noise. These were linked to a single hidden layer comprised of three units before final estimation by the output layer. For purposes of our model, we postulated these three units in the hidden layer as follows: (1) synaptic connectivity or neural synchrony between neurons via node $H(1: 1)$ that reflects the "processing capacity" factor of Polich context updating model, (2) speed of stimulus classification via node $\mathrm{H}(1: 2)$ that reflects the "attention allocation" factor of Polich's updating model, and (3) resource allocation demands via node $\mathrm{H}(1: 3)$ of "resource allocation" that reflects the "task demand" of Polich's model. As shown in Figure 3, the strongest synaptic weight was found between node $\mathrm{H}(1: 3)$ of resource allocation and P3AERP latency. Competing levels of noise had primarily an inhibitory effect on resource allocation, possibly reflecting the competition of neural resources engaged in $\mathrm{P} 300$ generation versus neural resources occupied by competing noise. The trend for longer latencies associated with increasing levels of competing noise reflected the mean data shown in Table 7.

3.4.1. Amplitude Results for P3AERP. The results of neural network analyses for P3AERP amplitude results are shown in Figure 4. For the three factors (groups, rate, and noise), 


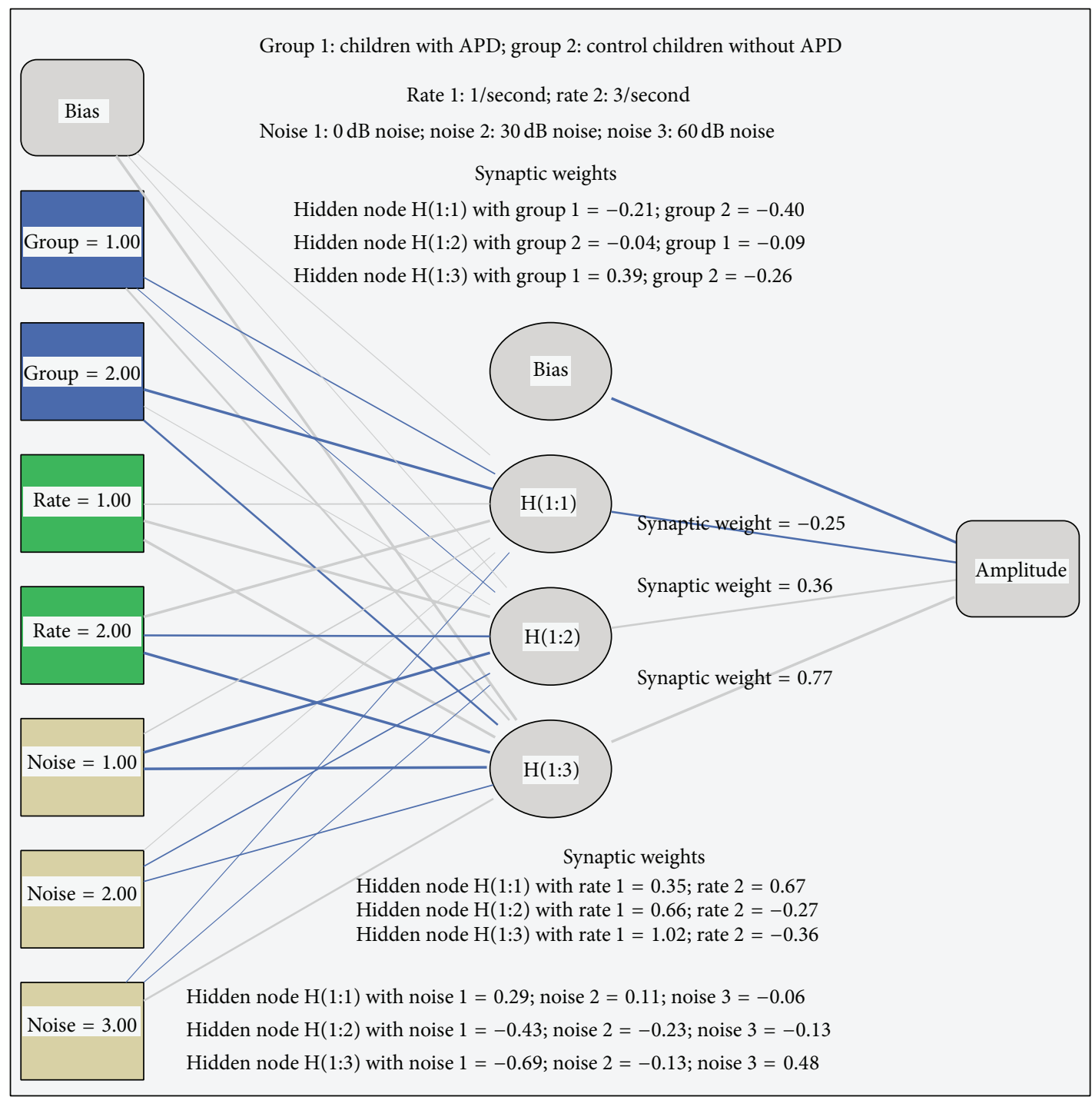

FIGURE 4

there were a total of seven units comprised of two units for groups, two units for rate, and three units for noise. These were linked to a single hidden layer comprised of three units before final estimation by the output layer. For purposes of our model, we postulated these two units in the hidden layer as follows: (1) strength of neural firing via node $H(1: 1)$, (2) attentional allocation via node $\mathrm{H}(1: 2)$, and (3) resource allocation via node $\mathrm{H}(1: 3)$. According to Polich [21], at least three factors (processing capacity, attention allocation, and task-related demands) control the neural generation of the P300 component. Based on this updating theory of the P300, we selected three nodes in the hidden layer for use in our study. The first node $\mathrm{H}(1: 1)$, that is, neural firing, was selected based on processing capacity. The second node $\mathrm{H}(1: 2)$ of stimulus classification was based on attention allocation, and the third node $H(1: 3)$, that is, resource allocation, was proposed based on task demands.

As shown in Figure 4, the strongest synaptic weight was observed between $\mathrm{H}(1: 3)$ node of resource allocation and
P3AERP amplitude. Competing levels of noise had primarily inhibitory influences on node $\mathrm{H}(1: 3)$, that is, resource allocation. Hence, it appears that, for both groups of children, competing noise decreased P3AERP amplitudes, possibly because of the associated reduction in neural resources available.

\section{Discussion}

4.1. P3AERP Latency Effects. The results of the current study indicate significantly longer P3AERP latencies for children with APD than for children without APD. Neural network analyses shown in Figure 3 indicated a strong association between speed of information processing and stimulusrelated factors in children with APD. These results appear to indicate that, for children with APD, P300 latency is significantly influenced by two factors (speed of information processing and task-related demands imposed by the rapid rates and competing noise). 
The longer P3AERP latencies in children with APD (relative to children without APD) found in our study have previously been attributed to slower speeds of information processing in children with APD. Studies by Jirsa [32] and Jirsa and Clontz [27] have shown differences in such fundamental auditory processing mechanisms across children with and without central auditory processing disorders. Children with APD showed significantly longer P3AERP latencies and smaller amplitudes than control children without APD on a binaural listening task [27]. Significant decreases in P3AERP latency and increase in P3AERP amplitudes have been shown in children with APD following therapeutic intervention [32].

Neural network modeling results of the current study suggest that reduction in allocation of neural resources can significantly inhibit P300 processing in children. P3AERP latency is considered to be an index of stimulus classification speed and is proportional to the time taken to detect and evaluate a target stimulus that is embedded in a stream of irrelevant stimuli [21].

4.2. P3AERP Amplitude Effects. There were no significant $(P>0.05)$ differences in P3AERP amplitude between groups (children with and without APD) but both groups showed significantly greater mean P3AERP amplitudes for the $1 / \mathrm{sec}$ condition than for the $3 / \mathrm{sec}$ condition, indicating that increasing the stimulus rate decreased neural processing resources at rapid rates. Neural network results shown in Figure 4 indicated a strong association between resource allocation and P3AERP amplitude. The relationship of P3AERP amplitudes with resource allocation in both groups can be explained by the triarchic model for P3AERP amplitude [30, 59, 60]. According to this model, three variables may make significant contributions to P3AERP amplitude: subjective probability of stimulus (P), stimulus meaning (M), and proportion of information transferred to subject $(\mathrm{T})$. It is possible that increasing stimulus rate in the current study influenced the subjective probability $(\mathrm{P})$ of the stimuli by increasing the temporal frequency of the target stimulus (hence reducing the novelty of the target stimulus) and reducing the P3AERP amplitude.

Neural network modeling results of our study indicate that, for both groups of school-age children, reduction in neural allocation of attention by competing noise was associated with smaller P3AERP amplitudes. P3AERP amplitude is believed to reflex the attentional resources needed for stimulus classification of target versus frequent stimuli [26]. Discriminating target or novel stimuli from frequent stimuli produce robust $\mathrm{P} 300$ responses that increase in amplitude as the probability of the target stimuli increases [21].

4.3. Stimulus-Related Effects. Analyses of variance results of this study (Tables 5 and 6) showed significant main effects of stimulus rate on P3AERP latency. Results of this study showed significantly greater mean P3AERP amplitudes for the $1 / \mathrm{sec}$ condition than for the $3 / \mathrm{sec}$ condition, indicating that increasing the stimulus rate decreased information processing resources at rapid rates. Neural network analyses in Figure 3 indicated that, for both groups, there was an inhibitory influence of competing noise on neural resource allocation, thereby increasing P3AERP latency and decreasing P3AERP amplitude.

Neural network modeling results in Figures 3 and 4 indicated that competing noise can limit resource allocation in school-age children with and without APD. The significant effects of stimulus rate on P3AERP latency and P3AERP amplitude in this study support findings of previous studies that showed significant effects of stimulus-related factors on P3AERP latency [14, 28, 61, 62]. Kilpeläinen et al. [61] showed significantly longer latencies and reduced amplitudes of P3AERP components for normally hearing children, when excessively long intervals occurred between target stimuli. Such effects on latency and amplitude were not seen in normally hearing adult listeners, suggesting differences in neural resources and processing for P3AERP generation between children and adults. Krishnamurti [28] measured P3AERP latencies in adults with APD and control adults without APD for tone burst stimuli presented in two conditions: (1) binaurally and (2) in conjunction with contralateral competing noise. Longer P3AERP latencies were found in adults with APD (compared to controls without APD) on both binaural and competing noise conditions. Also adults with APD showed longer P3AERP latencies on the contralateral competing noise than the binaural condition while control adults without APD showed no significant differences between P3AERP latencies in the binaural and competing conditions. McPherson and Salamat [14] studied the effects of varying ISI ( $1 \mathrm{sec}, 2 \mathrm{sec}$, and $4 \mathrm{sec}$ ) on P3AERP latency in 11 subjects with ADHD and 20 adult controls without ADHD. Subjects were required to respond by pushing a button for common stimuli presented and ignoring the rare stimuli. Significant differences between groups were found for each of the three ISIs. The control group showed significant differences in P3AERP latency across ISIs and the authors proposed that the longer P3AERP latencies with increasing ISI may be due to longer processing times needed to discriminate stimuli. In contrast, the group with $\mathrm{ADHD}$ showed no significant differences in P3AERP latency across ISIs, indicating reduced attention across all ISIs. Salamat and McPherson [62] studied the effects of varying interstimulus intervals (1 sec, $2 \mathrm{sec}$, $4 \mathrm{sec}$ ) on P3AERPs in 20 normally hearing adult listeners. P3AERP latencies were found to increase with increasing interstimulus interval (ISI) and the authors hypothesized that the longer P3AERP latencies may reflect the decline in attention and cognitive processing associated with longer ISIs.

The significant effects of stimulus rate on P3AERP latency and P3AERP amplitude in this study also question the "endogenous" nature of P3AERPs. By definition, P3AERPs are typically considered to be endogenous potentials that are influenced more by internal (subject-related) factors than external (stimulus-related) factors [8, 15, 63-65]. However, several studies have also shown that stimulus-related factors (e.g., frequency and intensity) can significantly influence latency and amplitude of P3AERPs [16, 18]. Increasing stimulus intensity will result in an increase in P3AERP amplitude and decrease P3AERP latency [18]. Polich et al. [18] showed that, above $75 \mathrm{~dB}$ SPL, the amplitude of the P3AERP does not significantly increase, indicating that the 
exogenous component is maximized. If P3AERP truly represents an endogenous potential, the P3AERP amplitudes and latencies should be similar at suprathreshold and threshold levels. P3AERP waveforms have been shown to be larger in amplitude and shorter in latency at suprathreshold levels (75 dB SPL) compared to threshold levels [16]. In the current study, P3AERP amplitudes were found to be reduced with competing noise, reflecting that there may be more than just an endogenous aspect to components of the P3AERPs.

\section{Conclusions}

Results of the current study offer promise for use of P3AERPs in evaluation of auditory processing disorders in school-age children. More research is needed, however, before the use of P3AERPs in a standard APD battery can be advocated for school-age children. The utility of other physiological measures of brainstem processing has already been demonstrated in investigating auditory training (plasticity) effects [66, 67]. More research on neural correlates of cortical processing by measures such as P3AERPs will provide insight into the listening skills of school-age children.

\section{Conflict of Interests}

The author declares that there is no conflict of interests regarding the publication of this paper.

\section{Acknowledgments}

Assistance provided by students Julie Ann Embry, Mary Young, and Chassity South during the study is highly appreciated.

\section{References}

[1] American-Speech-Language-Hearing Association (ASHA), (Central) Auditory Processing Disorders-The Role of the Audiologist. [Position Statement], American-Speech-LanguageHearing Association, 2005, http://www.asha.org/policy.

[2] J. Jerger and F. Musiek, "Report of the consensus conference on the diagnosis of auditory processing disorders in school-aged children," Journal of the American Academy of Audiology, vol. 11, no. 9, pp. 467-474, 2000.

[3] S. Rosen, M. Cohen, and I. Vanniasegaram, "Auditory and cognitive abilities of children suspected of auditory processing disorder (APD)," International Journal of Pediatric Otorhinolaryngology, vol. 74, no. 6, pp. 594-600, 2010.

[4] A. T. Cacace and D. J. McFarland, "The importance of modality specificity in diagnosing central auditory processing disorder," Journal of the American Academy of Audiology, vol. 14, no. 2, pp. 112-113, 2005.

[5] A. T. Cacace and D. J. McFarland, "Factors influencing tests of auditory processing: a perspective on current issues and relevant concerns," Journal of the American Academy of Audiology, vol. 24, no. 7, pp. 572-589, 2013.

[6] F. E. Musiek, T. J. Bellis, and G. D. Chermak, "Nonmodularity of the central auditory nervous system: implication for (central) auditory processing disorder," Journal of the American Academy of Audiology, vol. 14, no. 2, pp. 128-138, 2005.
[7] D. R. Moore and M. A. Ferguson, "It is neither necessary nor desirable to test for abnormalities in other modalities when diagnosing auditory processing disorder (APD)," Journal of the American Academy of Audiology, vol. 25, no. 7, pp. 695-696, 2014.

[8] J. Butcher, "Cognitive auditory responses," in Principles and Applications in Auditory Evoked Potentials, J. T. Jacobson, Ed., Allyn \& Bacon, Boston, Mass, USA, 1992.

[9] G. D. Chermak and F. E. Musiek, Central Auditory Processing Disorders: New Perspectives, Singular, San Diego, Calif, USA, 1997.

[10] F. E. Musiek, "Habilitation and management of auditory processing disorders," Journal of the American Academy of Audiology, vol. 10, pp. 329-342, 1999.

[11] F. E. Musiek and S. Bornstein, "Auditory event-related potentials in central auditory disorders in young children," in Central Auditory Processing: A Transdisciplinary View, J. Katz, N. A. Stecker, and D. Henderson, Eds., Mosby, St. Louis, Mo, USA, 1994.

[12] J. Polich, L. Howard, and A. Starr, "P300 latency correlates with digit span," Psychophysiology, vol. 20, no. 6, pp. 665-669, 1983.

[13] J. Polich, "Attention, probability, and task demands as determinants of P300 latency from auditory stimuli," Electroencephalography \& Clinical Neurophysiology, vol. 63, no. 3, pp. 251-259, 1986.

[14] D. L. McPherson and M. T. Salamat, "Interactions among variables in the P300 response to a continuous performance task in normal and ADHD adults," Journal of the American Academy of Audiology, vol. 15, no. 10, pp. 666-677, 2004.

[15] J. W. Hall, Handbook of Auditory Evoked Responses, Allyn \& Bacon, Boston, Mass, USA, 1992.

[16] F. E. Musiek, R. Froke, and J. Weihing, "The auditory P300 at or near threshold," Journal of the American Academy of Audiology, vol. 16, no. 9, pp. 699-708, 2005.

[17] J. Polich, "Habituation of P300 from auditory stimuli," Psychobiology, vol. 17, pp. 19-28, 1989.

[18] J. Polich, P. C. Ellerson, and J. Cohen, "P300, stimulus intensity, modality, and probability," International Journal of Psychophysiology, vol. 23, no. 1-2, pp. 55-62, 1996.

[19] W. T. Roth, G. H. Blowers, C. M. Doyle, and B. S. Kopell, "Auditory stimulus intensity effects on components of the late positive complex," Electroencephalography and Clinical Neurophysiology, vol. 54, no. 2, pp. 132-146, 1982.

[20] E. Donchin, “Surprise!...Surprise?” Psychophysiology, vol. 18, no. 5, pp. 493-513, 1981.

[21] J. Polich, "Updating P300: an integrative theory of P3a and P3b," Clinical Neurophysiology, vol. 118, no. 10, pp. 2128-2148, 2007.

[22] J. S. Buchwald, "Comparison of plasticity in sensory and cognitive processing systems," Clinics in Perinatology, vol. 17, no. 1, pp. 57-66, 1990.

[23] D. McPherson, Late Potentials of the Auditory System, Singular, San Diego, Calif, USA, 1996.

[24] J. W. Tampas, A. W. Harkrider, and M. S. Hedrick, "Neurophysiological indices of speech and nonspeech stimulus processing," Journal of Speech, Language, and Hearing Research, vol. 48, no. 5, pp. 1147-1164, 2005.

[25] D. C. Emanuel, "The auditory processing battery: survey of common practices," Journal of the American Academy of Audiology, vol. 13, no. 2, pp. 93-117, 2002.

[26] C. C. Duncan-Johnson and E. Donchin, "The time constant in P300 recording," Psychophysiology, vol. 16, no. 1, pp. 53-55, 1979. 
[27] R. E. Jirsa and K. B. Clontz, "Long latency auditory event-related potentials from children with auditory processing disorders," Ear and Hearing, vol. 11, no. 3, pp. 222-232, 1990.

[28] S. Krishnamurti, "P300 auditory event-related potentials in binaural and competing conditions in adults with central auditory processing disorders," Contemporary Issues in Communication Sciences Disorders, vol. 28, pp. 40-47, 2001.

[29] F. E. Musiek, J. A. Baran, and M. L. Pinheiro, "P300 results in patients with lesions of the auditory areas of the cerebrum," Journal of the American Academy of Audiology, vol. 3, no. 1, pp. 5-15, 1992.

[30] R. Johnson Jr., "The amplitude of the P300 component of the event-related potential: review and synthesis," in Advances in Psychophysiology, P. K. Ackles, J. R. Jennings, and M. G. H. Coles, Eds., vol. 3, pp. 69-137, Jai Press, Greenwich, Conn, USA, 1988.

[31] J. Polich, "P300, probability, and interstimulus interval," Psychophysiology, vol. 27, no. 4, pp. 396-403, 1990.

[32] R. E. Jirsa, "The clinical utility of the P3AERP in children with auditory processing disorders," Journal of Speech and Hearing Research, vol. 35, no. 4, pp. 903-912, 1992.

[33] S. Krishnamurti, "Monaural low redundancy speech tests," in Handbook of Central Auditory Processing Disorders, F. E. Musiek and G. D. Chermak, Eds., Plural, San Diego, Calif, USA, 2007.

[34] S. Krishnamurti, "Pathways: Role of P300 auditory eventrelated potentials in evaluating auditory processing disorders," The Hearing Journal, vol. 58, no. 7, article 49, 2005.

[35] H. Abdi, "Neural networks," in Encyclopedia of Social Sciences Research Methods, Sage, Thousand Oaks, Calif, USA, 2003.

[36] R. C. Gismondi, R. M. V. R. Almeida, and A. F. C. Infantosi, "Artificial neural networks for infant mortality modelling," Computer Methods and Programs in Biomedicine, vol. 69, no. 3, pp. 237-247, 2002.

[37] Y.-C. Li, L. Liu, W.-T. Chiu, and W.-S. Jian, "Neural network modeling for surgical decisions on traumatic brain injury patients," International Journal of Medical Informatics, vol. 57, no. 1, pp. 1-9, 2000.

[38] S. Yamamura, "Clinical application of artificial neural network (ANN) modeling to predict pharmacokinetic parameters of severely ill patients," Advanced Drug Delivery Reviews, vol. 55, no. 9, pp. 1233-1251, 2003.

[39] S. Krishnamurti, L. Drake, and J. King, "Neural network modeling of central auditory dysfunction in Alzheimer's disease," Neural Networks, vol. 24, no. 6, pp. 646-651, 2011.

[40] A. Keshavarzi and F. Sarmadian, "Comparison of artificial neural network and multivariate regression methods in prediction of soil cation exchange capacity," International Journal of Environmental and Earth Sciences, vol. 15, no. 2, pp. 167-174, 2010.

[41] American National Standards Institute, "American national standard criteria for specifications for audiometers," Tech. Rep. S3.6-1996, ANSI, New York, NY, USA, 1996.

[42] American National Standards Institute, "Maximum permissible ambient noise for audiometric test rooms," ANSI S3.1-1999, ANSI, New York, NY, USA, 1999.

[43] L. Fisher, Fisher's Auditory Problems Checklist, Grant Wood, Cedar Rapids, Iowa, USA, 1980.

[44] R. W. Keith, SCAN: A Test for Auditory Processing Disorders in Children, The Psychological Corporation, San Antonio, Tex, USA, 1986.
[45] R. W. Keith, SCAN-A: A Test for Auditory Processing Disorders in Adolescents and Adults, The Psychological Corporation, San Antonio, Tex, USA, 1992.

[46] R. L. Schow and G. D. Chermak, "Implications from factor analysis for central auditory processing disorders," American Journal of Audiology, vol. 8, no. 2, pp. 137-142, 1999.

[47] D. M. Domitz and R. L. Schow, "A new CAPD batterymultiple auditory processing assessment: factor analysis and comparisons with SCAN," American Journal of Audiology, vol. 9, no. 2, pp. 101-111, 2000.

[48] M. Pinheiro, "Tests of central auditory function for children with learning disabilities," in Central Auditory Dysfunction, R. W. Keith, Ed., pp. 223-256, Grune \& Stratton, New York, NY, USA, 1977.

[49] F. E. Musiek, "Frequency (pitch) and duration pattern tests," Journal of the American Academy of Audiology, vol. 5, no. 4, pp. 265-268, 1994.

[50] F. E. Musiek and M. L. Pinheiro, "Frequency patterns in cochlear, brainstem, and cerebral lesions," Audiology, vol. 26, no. 2, pp. 79-88, 1987.

[51] F. E. Musiek, J. A. Baran, and M. L. Pinheiro, "Duration pattern recognition in normal subjects and patients with cerebral and cochlear lesions," Audiology, vol. 29, no. 6, pp. 304-313, 1990.

[52] T. J. Bellis, Assessment and Management of Central Auditory Processing Disorders: From Science to Practice, ThompsonDelmar Learning, 2nd edition, 2003.

[53] L. G. Wall, S. A. Davidson, and S. D. Dalebout, "Determining latency and amplitude for multiple peaked P300 waveforms," Journal of the American Academy of Audiology, vol. 2, no. 3, pp. 189-194, 1991.

[54] S. D. Dalebout and R. R. Robey, "Comparison of the intersubject and intrasubject variability of exogenous and endogenous auditory evoked potentials," Journal of the American Academy of Audiology, vol. 8, no. 5, pp. 342-354, 1997.

[55] G. Dawson, C. Finley, S. Phillips, and A. Lewy, "A comparison of hemispheric asymmetries in speech-related brain potentials of autistic and dysphasic children," Brain and Language, vol. 37, no. 1, pp. 26-41, 1989.

[56] J. Polich, J. E. Alexander, L. O. Bauer et al., "P300 topography of amplitude/latency correlations," Brain Topography, vol. 9, no. 4, pp. 275-282, 1997.

[57] D. E. Rumelhart, J. L. McClelland, and The PDP Research Group, Parallel Distributed Processing: Explorations in the Microstructure of Cognition, vol. 1: Foundations, MIT Press, Cambridge, Mass, USA, 1986.

[58] B. Widrow and M. A. Lehr, "30 years of adaptive neural networks: perceptron, madaline, and backpropagation," Proceedings of the IEEE, vol. 78, no. 9, pp. 1415-1442, 1990.

[59] R. Johnson Jr., “A triarchic model of P300 amplitude," Psychophysiology, vol. 23, no. 4, pp. 367-384, 1986.

[60] R. Johnson Jr., "On the neural generators of the P300 component of the event-related potential," Psychophysiology, vol. 30, no. 1, pp. 90-97, 1993.

[61] R. Kilpeläinen, A. Koistinen, M. Könönen, E. Herrgård, J. Partanen, and J. Karhu, "P300 sequence effects differ between children and adults for auditory stimuli," Psychophysiology, vol. 36, no. 3, pp. 343-350, 1999.

[62] M. T. Salamat and D. L. McPherson, "Interactions among variables in the P300 response to a continuous performance task," Journal of the American Academy of Audiology, vol. 10, no. 7, pp. 379-387, 1999. 
[63] J. M. Stapleton, T. O'Reilly, and E. Halgren, "Endogenous potentials evoked in simple cognitive tasks: scalp topography," International Journal of Neuroscience, vol. 36, no. 1-2, pp. 75-87, 1987.

[64] S. Sutton, M. Braren, and J. Zubin, "Evoked-potential correlates of stimulus uncertainty," Science, vol. 150, no. 3700, pp. 11871188, 1965.

[65] S. Sutton, P. Tueting, J. Zubin, and E. R. John, "Information delivery and the sensory evoked potential," Science, vol. 155, no. 3768, pp. 1436-1439, 1967.

[66] N. M. Russo, T. G. Nicol, S. G. Zecker, E. A. Hayes, and N. Kraus, "Auditory training improves neural timing in the human brainstem," Behavioural Brain Research, vol. 156, no. 1, pp. 95103, 2005.

[67] S. Krishnamurti, J. Forrester, C. Rutledge, and G. W. Holmes, "A case study of the changes in the speech-evoked auditory brainstem response associated with auditory training in children with auditory processing disorders," International Journal of Pediatric Otorhinolaryngology, vol. 77, no. 4, pp. 594-604, 2013. 

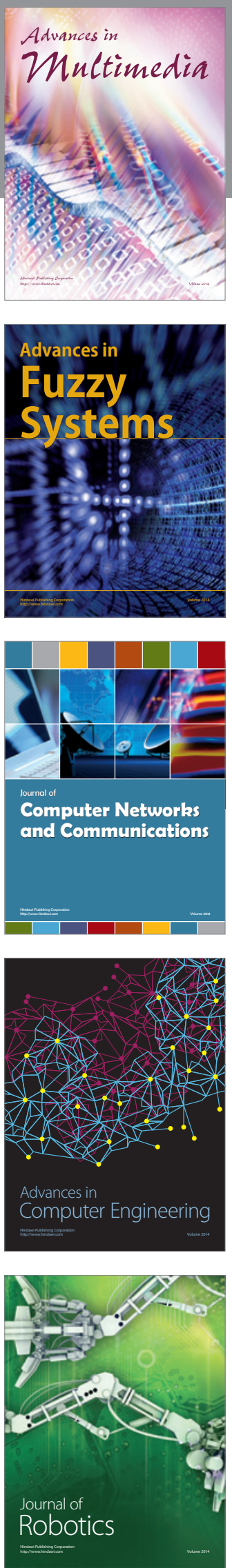

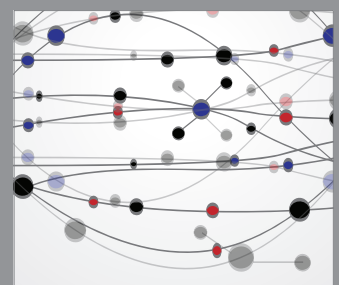

The Scientific World Journal
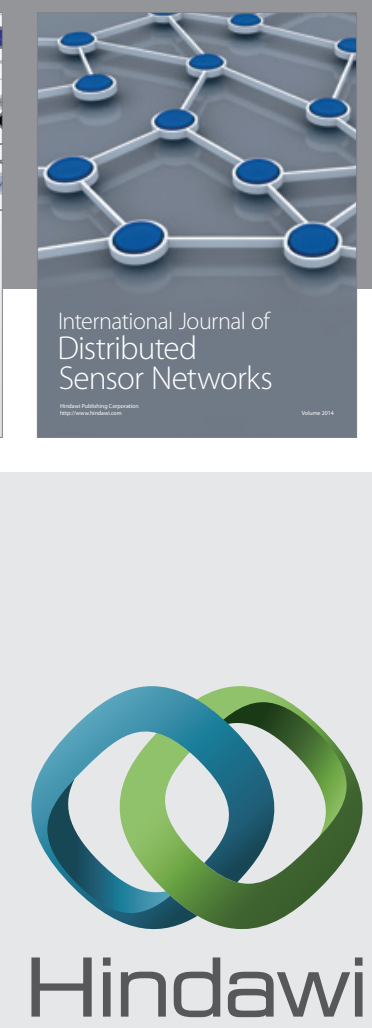

Submit your manuscripts at

http://www.hindawi.com
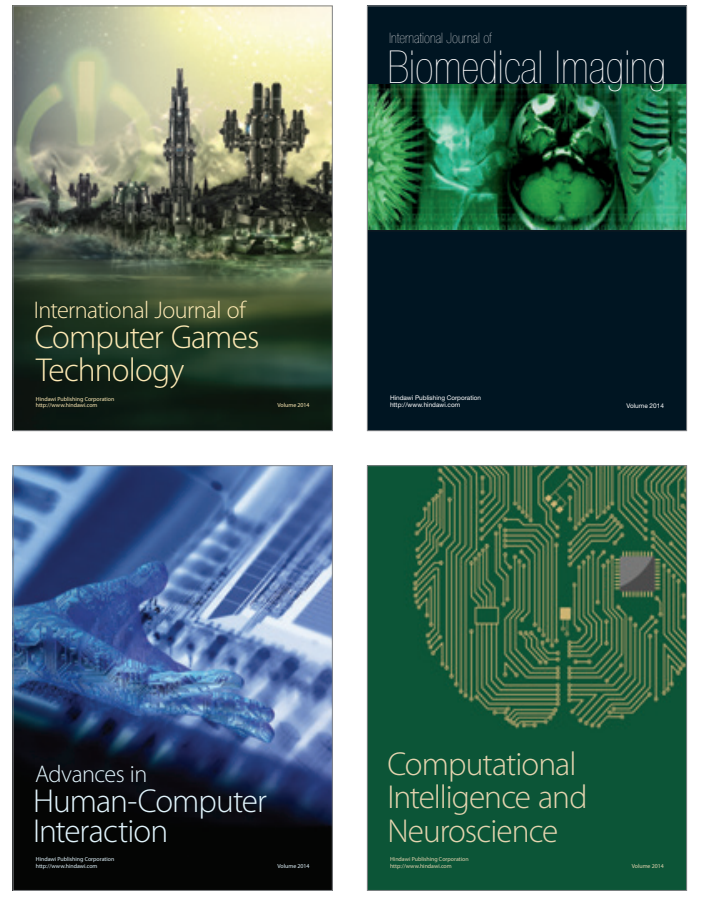
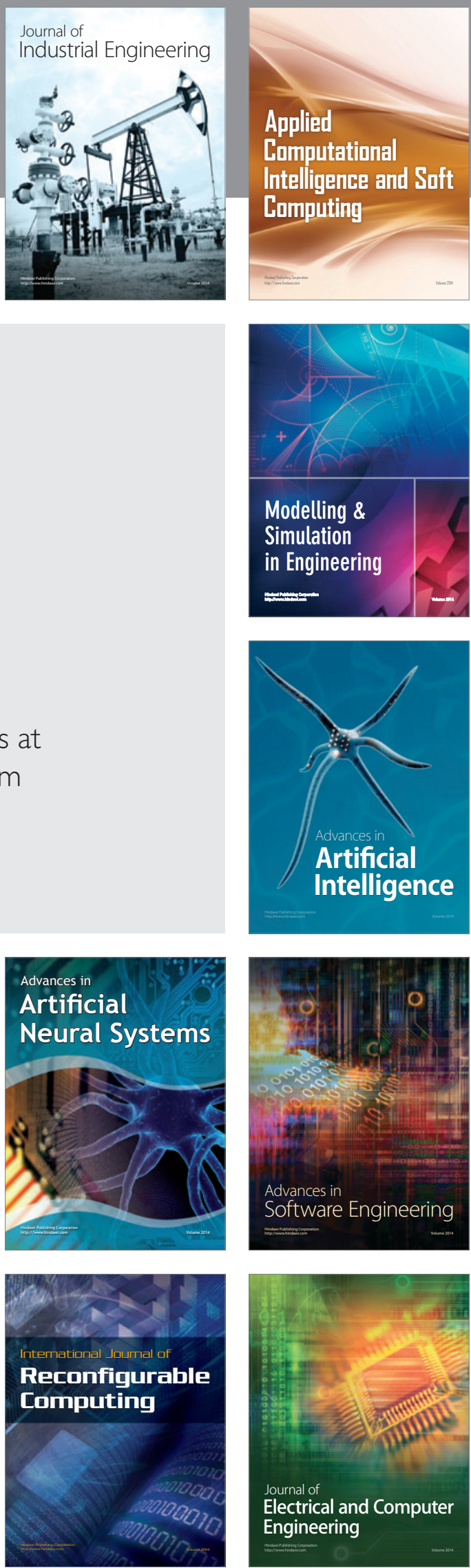